\title{
ON DYNAMIC KIEFER-WOLFOWITZ \\ STOCHASTIC APPROXIMATION PROCEDURES
}

El Sayed Sorour

Military Technical College,

Department of Mathematics.

\section{Abstarct}

Let $M(x)$ be a function from $R^{k} \rightarrow R$. Let $\theta_{n}, n=1,2, \ldots$ be vectors in $\mathrm{R}^{\mathrm{k}}, \theta_{1}$ being the value at which $M(x)$ achieves its unique minimum. Set $M(x)=M_{1}(x)$, for $n=1,2, \ldots$, set $M_{n}(x)=$ $M\left(x-\theta_{n}-\theta_{l}\right)$. Then $\theta_{n}$ is the unique minimum of $M_{n}(x)$, which is unknown and is to be estimated. In our case, we assume that $\theta_{n}$ moves in such a manner that $\theta_{n+1}=g_{n}\left(\theta_{n}\right)+v_{n}$ where $g_{n}\left(\theta_{n}\right)$ is general non-linear k-vector measurable function (known) defined for all $\mathrm{x} \in \mathrm{R}^{\mathrm{k}}$ and $\mathrm{v}_{\mathrm{n}}$ is an unknown $\mathrm{k}$-vector function ( $\mathrm{random}$ or non-random) independent of $x$. Let $a_{n}, c_{n}, n=1,2, \ldots$ be two sequences of positive numbers. Let $x_{1}$ be an arbitrary random variable. Define for $n=1,2, \ldots, x_{n+1}=\stackrel{*}{x}_{n}+a_{n}\left(\stackrel{*}{y}_{2 n}-\stackrel{*}{y}_{2 n-1}\right) / c_{n}$ where $\stackrel{*}{x}_{n}=g_{n}\left(x_{n}\right)$, and $\stackrel{*}{y}_{2 n}, \stackrel{*}{y}_{2 n-1}$ are random variables such that their expectations given $x_{1}, x_{2}, \ldots \ldots x_{n}$ are $\sum_{i=1}^{k} M_{n+1}\left(x_{n}+e_{i} c_{n}\right) e_{i}$ and $\sum_{i=1}^{k} M_{n+1}\left(x_{n}-e_{i} c_{n}\right) e_{i}$ respectively and their conditional variance are bounded by a constant $\sigma^{2}$ and they are conditionally independent. Under conditions similar to those used by Dupac (1966), we show that $\left\|x_{n}-\theta_{n}\right\| \rightarrow 0$ with probability one. 


\section{INTRODUCTION}

This paper is concerned with the dynamic kiefer-Wolfowitz (KW) stochastic approximation procedure. This problem has been firstly studied by Dupac [2], [3]. He discussed in his papers the cases where the movement of the maximum can be expressed by a certain linear function of its present location and determinestic trend, i.e. he assume that

$$
\theta_{n+1}=\left(1+\frac{1}{2}\right) \theta_{n}+v_{n}
$$

where $\Theta_{n}$ is the unique maximum of the regression function $M_{n}(x)$. Uosaki, K. (1974) discussed the one-dimensional dynamic Rolbins-Morro (rii) process. He considers the case where the movement of the root can be expressed by a specified non-linear function oE its present location. Sorour (1978) geenralizes Worais iesult to the multidimersional dynamic (Re). In this paper; we shall be concerned with the non-linear multidimensional dynamic systems. The convergence of the approximation to the moving minimum of a nonlinear regression function, with probability one, is proved. In sec. 4 we prove the convergence with probability one and in sec. '5, we show that under some regularity conditions on the noise, the process is asymtotoically normal.

\section{DESCRIPTION OF THE PROCEDURE}

Let $\mathrm{R}^{\mathrm{k}}$ be a real $\mathrm{k}$-dimensional vector space. If $\mathrm{x}$ and $y$ are two vectors in $\mathrm{R}^{\mathrm{k}}$ we denote their-inner product by $(\mathrm{x}, \mathrm{y})$ and their nroms by $\|x\|$ and $\|y\|$ respectively. Let $M_{n}(x)$ be a (unknown) 
function from $\mathrm{R}^{\mathrm{k}} \rightarrow \mathrm{R}$. Assume that $\theta_{\mathrm{n}}$ is the unique minimum of $M_{n}(x)$. Our goal is to estimate $\theta_{n}$. In our case we assume that $\theta_{n}$ moves in à manner that

$$
\theta_{n+1}=g_{n}\left(\theta_{n}\right)+v_{n}
$$

where $g_{n}(x)$ is in general a nonlinear measurable function (known) from $\mathrm{R}^{\mathrm{k}} \rightarrow \mathrm{R}^{\mathrm{k}}$ and $\mathrm{v}_{\mathrm{n}}$ is unknown $\mathrm{k}$ vector (non-random).

Let $a_{n}, n=1,2, \ldots$ be positive numbers. Let $x_{1}$ be an arbitrary random variable define for $n=1,2, \ldots$

$$
x_{n+1}=\stackrel{*}{x}_{n}-a_{n} F_{n}
$$

where

$$
\begin{aligned}
& \stackrel{\star}{\mathrm{x}}_{\mathrm{n} 1}=\mathrm{g}_{\mathrm{n}}\left(\mathrm{x}_{\mathrm{n}}\right) ; \\
& \mathrm{F}_{\mathrm{n} 1}=\overline{\mathrm{D}}_{\mathrm{n}}+\mathrm{d}_{\mathrm{n}}
\end{aligned}
$$

and the ith compenent of $\bar{D}_{n}$ is given by

$$
\bar{E}_{n}^{i}=\left[M_{n+1}\left(x_{n}+e_{i} c_{n}\right)-M_{n+1}\left(x_{n}-e_{i} c_{n}\right)\right] / 2 c_{n} \text {. }
$$

where $e_{i}$ is the $i$ th column in the identity matrix and $c_{n} i s$ a positive sequence of real numbers. Let ${ }^{\Im} n$ be the $\sigma-f i e l d$ generated by $x_{1}, x_{2}, \ldots, x_{n}$. For $x$ a random vector in $R^{k}$ let $E_{n}(x)$ and $\operatorname{var}_{n}(x)$ denote the conditional expectation and the conditional variance of $x$ with respect to ${ }^{*}$ respectively. Let

$$
E_{n}\left(d_{n}\right)=0
$$

and for constant $k_{1}$ let

$$
E_{n}\left(\left\|d_{n}\right\|^{2}\right) \leq k_{1} \quad c_{n}^{-2}
$$

Let $D_{n}(x)$ denotes the vector of the partial derivatives of $M_{n}(x)$ Then

$$
D_{n}^{i}(x)=\frac{\partial M_{n}(x)}{\partial x^{i}}
$$


Assume that

$$
\left\|D_{n}-D_{n+1}\left(\stackrel{*}{x}_{n}\right)\right\| \leq k_{2} c_{n}
$$

In what follows ( $Q, \widetilde{F}, P$ ) will be a probability space, relations and convergence of random variables, vectors and matrices will be meant with probability one. The indicator function of set $A$ will be denoted $x^{A}=R^{k \times k}$ is the space of all real $k \times k$ natrices. The unit matrix in $\mathrm{R}^{\mathrm{k} \times \mathrm{k}}$ is denoted by $\mathrm{I}$ and $\|\cdot\|$ is the Euclidean norm. With $h_{n}$ a sequence of numbers let $O\left(h_{n}\right), O\left(h_{n}\right)$ denote sequerices $g_{n}$ and $G_{n}$, say of elements in one of the sets $R, R^{k}$ such that $h_{\mathrm{n}}^{-1} \mathrm{~g}_{\mathrm{n}} \rightarrow 0,\left\|\mathrm{~h}_{\mathrm{n}}^{-1} \mathrm{C}_{\mathrm{n}}\right\| \leq \mathrm{f}$ for $\mathrm{f} \in \mathrm{R}$.

Remark : Let $Y_{n}(i, 1), Y_{n}(i, 2)$ be two random variables such

$$
\begin{aligned}
& E_{n}\left(Y_{n}(i, 1)\right)=M_{n+1}\left(\stackrel{\star}{X}_{n}+e_{i} c_{n}\right) \\
& E_{n 1}\left(Y_{n}(i, 2)\right)=M_{n+1}\left(\stackrel{x}{X}_{n}-e_{i} c_{n}\right)
\end{aligned}
$$

Then $F_{n}=\left[Y_{n}(i, 1)-Y_{n}(i, 2)\right] / 2 c_{n}$ and $Y_{n}(i, 1)$ and $Y_{n}(i, 2)$ are conditionaly independent. If $\operatorname{Var}{ }_{n}\left(Y_{n}(i, l)\right)$ and $\operatorname{Var}\left(x_{n}(i, 2)\right)$ are bounded. Then (2.5) holed. Also let $H_{n}(x)$ be the Hessian of $M_{n}(x)$ i.e.

$$
H_{n}^{i j}(x)=\frac{\partial^{2} M_{n}(x)}{\partial x^{i} \partial x^{j}}
$$

If $\sup _{x}\left\{\left\|H_{n}(x)\right\|<\infty\right\}$ for $n=1,2, \ldots$ Then (2.6) holds.

\section{CONSITIONS}

Conditions on the regression function $M_{n}(x)$ Ml : There existy two numbers $A$ and $B$ such that

$$
\left\|D_{n}(x)\right\| \leq A\left\|x-\theta_{n}\right\|+B
$$

M2 : For all $\delta>0$, we have 


$$
\operatorname{if}_{n \in N} \quad \delta\left\langle\left\|x-\theta_{n}\right\|<\delta^{-1}\left\langle x-\theta_{n}, D_{n}(x)\right\rangle>0\right.
$$

Conditions on the sequences $a_{n}$ and $c_{n}$

Al : $\quad \sum_{n=1}^{\infty} a_{n} c_{n}<\infty ; \quad \sum_{n=1}^{\infty} a_{n}=\infty, \quad \sum a_{n}^{2}<\infty, \quad \sum_{n=1}^{\infty} a_{n}^{2} c_{n}^{-2}<\infty$ Conditions on the functions $g_{n}(x)$ and $v_{n}$

Gl : There exists a sequence of positive number $\gamma_{n}$ independent of $x$ and $y$ such that

$$
\left\|g_{n}(x)-g_{n}(x)\right\| \leq \gamma_{n}\|x-y\| \text { for all } x, y \in R^{k} \text {. }
$$

G2 : $\sum_{n=1}^{\infty}\left(\gamma_{n}^{2}-1\right)^{+}<\infty$; where $z^{+}$means $(x+|z|) / 2$

G3 $\lim _{n \rightarrow \infty} g_{n}(x)-g_{n}(y)-v_{n}$ exists for all $\|x-y\|<\infty$ G4: $\sum_{n=1}^{\infty}\left\|v_{n}\right\|<\infty$

\section{ASYMPTOTIC CONVERGENCE}

Theorem 4.1. If the conditions Ml, M2, Al, Gl-G4 hold. Then

$$
\lim _{\mathrm{n} \rightarrow \infty}\left\|\mathrm{x}_{\mathrm{n}}-\theta_{\mathrm{n}}\right\|=0
$$

Proof :- From (2.1) and (2.2) we have

$$
x_{n+1}-\theta_{n+1}=g_{n}\left(x_{n}\right)-g_{n}\left(\theta_{n}\right)-v_{n}-a_{n} F_{n}
$$

Thus

$$
\begin{aligned}
\left\|x_{n+1}-\theta_{n+1}\right\|^{2}= & \left\|g_{n}\left(x_{n}\right)-g_{n}\left(\theta_{n}\right)-v_{n}\right\|^{2}-2 a_{n}\left\langle g_{n}\left(x_{n}\right)-g_{n}\left(\theta_{n}\right)\right. \\
& \left.-v_{n}, F_{n}\right\rangle+a_{n}^{2}\left\|F_{n}\right\|^{2}, \\
= & \left\|g_{n}\left(x_{n}\right)-g_{n}\left(\theta_{n}\right)\right\|^{2}-2\left\langle g_{n}\left(x_{n}\right)-g_{n}\left(\theta_{n}\right), v_{n}\right\rangle+ \\
& +\|v\|^{2}-2 a_{n}\left\langle\stackrel{x}{x}_{n}-\theta_{n+1}, F_{n}\right\rangle+a_{n}^{2}\left\|F_{n}\right\|^{2}
\end{aligned}
$$

Using (2.4) we obtain 


$$
\begin{aligned}
E_{n}\left(\left\|x_{n+1}-\theta_{n+1}\right\|^{2}\right)= & \left\|g_{n}\left(x_{n}\right)-g_{n}\left(\theta_{n}\right)\right\|^{2}-2\left\langle g_{n}\left(x_{n}\right)-g_{n}\left(\theta_{n}\right), v_{n}\right\rangle \\
& +\left\|v_{n}\right\|^{2}-2 a_{n}\left\langle x_{n}^{*}-\theta_{n+1} ; \bar{D}_{n}\right\rangle+a_{n}^{2} E_{n}\left(\left\|F_{n}\right\|^{2}\right) \\
\leq & \left\|g_{n}\left(x_{n}\right)-g_{n}\left(\theta_{n}\right)\right\|^{2}-2 a_{n}\left\langle\dot{x}_{n}^{*}-\theta_{n+1} ; \bar{D}_{n+1}\left(\stackrel{x}{x}_{n}\right)\right\rangle \\
& +o\left(\left\|v_{n}\right\|\left\|g_{n}\left(x_{n}\right)-g_{n}\left(\theta_{n}\right)\right\|\right)+o\left(\left\|v_{n}\right\|^{2}\right. \\
& +o\left(a_{n}\left\|g_{n}\left(x_{n}\right)-g_{n}\left(\theta_{n}\right)-v_{n}\right\|\left\|\bar{D}_{n}-D_{n+1}\left(\stackrel{x}{n}_{n}\right)\right\|^{2}\right) \\
& +o\left(a_{n}^{2}\left\|D_{n+1}\left(\stackrel{x}{x}_{n}^{*}\right)\right\|^{2}\right)+o\left(a_{n}^{2}\left\|\bar{D}_{n}-D_{n+1}\left(\stackrel{x}{n}_{n}\right)\right\|^{2}\right) \\
& +o\left(a_{n}^{2}\left\|E_{n}\left(d_{n}^{2}\right)\right\|\right)
\end{aligned}
$$

By (2.5), 92.6) and $M l$ and using the identity $\|x\| \leq\|x\|^{2}+1$, we obtain

$$
\begin{aligned}
E_{n}\left(\left\|x_{n+1}-\theta_{n+1}\right\|^{2}\right)= & \left\|g_{n}\left(x_{n}\right)-g_{n}\left(\theta_{n}\right)\right\|^{2}-\left(1+0\left\|v_{n}\right\|\right)+o\left(a_{n} c_{n}\right) \\
& \left.+o\left(a_{n}^{2}\right)\right)-2 a_{n}\left\langle\stackrel{x}{x}_{n}-\theta_{n+1} ; D_{n+1}\left(\stackrel{x}{n}_{n}\right)\right\rangle \\
& +o\left(\left\|v_{n}\right\|+\left\|v_{n}\right\|^{2}+a_{n} c_{n}+\left\|v_{n}\right\| a_{n} c_{n}+a_{n}^{2}\right) \\
= & \left\|g_{n}\left(x_{n}\right)-g_{n}\left(\theta_{n}\right)\right\|^{2}\left(1+\mu_{n}\right) \\
& -2 a_{n}\left\langle\stackrel{x}{x}_{n}-\theta_{n+1} ; D_{n+1}\left(\stackrel{*}{x}_{n}\right)\right\rangle+\delta_{n} \quad(4.2)
\end{aligned}
$$

where

$$
\begin{aligned}
& \mu_{n}=o\left(\left\|v_{n}\right\|+a_{n} c_{n}+a_{n}^{2}\right) \\
& \delta_{n}=O\left(\mu_{n}+a_{n}^{2} c_{n}^{-2}\right)
\end{aligned}
$$

From $A l$ and G4 it follows that

$$
\sum_{n=1}^{\infty} \mu_{n}<\infty \quad \text { and } \quad \sum_{n=1}^{\infty} \delta_{n}<\infty
$$

Using Gl, (4.2) can be written as 


$$
\begin{aligned}
E_{n}\left(\left\|x_{n+1}-\theta_{n+1}\right\|^{2}\right) \leq & \left\|x_{n}-\theta_{n}\right\|^{2}-\left(1+\mu_{n}+\left(\gamma_{n}^{2}-1+1\right)\right. \\
& +2 a_{n}\left\langle x_{n}^{*}-\theta_{n+1} ; D_{n+1}\left(\stackrel{x}{x}_{n}\right)\right\rangle+\delta_{n}
\end{aligned}
$$

From M2, G2, (4.3) and (4.4), it follows from Theorem 1 Robbins and siegmund [8] that

$$
\lim _{\mathrm{n} \rightarrow \infty}\left\|\mathrm{x}_{\mathrm{n}}-\theta_{\mathrm{n}}\right\| \text { exists and finite }
$$

and

$$
\sum_{n=1}^{\infty} a_{n}\left\langle\stackrel{*}{x}_{n}-\theta_{n+1} ; D_{n+1}\left(\stackrel{*}{x}_{n}\right)\right\rangle\langle\infty .
$$

Using $M 2, A l$ and $G 3$ obtain that

$\lim _{n \rightarrow \infty}\left\|x_{n}-\theta_{n}\right\|=0$, which completes the proof of theorem.

\section{ASYMPTOTIC NORMALITY OF THE PROCEDURE}

Theorem 5.1. L.et $A ; \mathrm{P} \in \mathrm{R}^{\mathrm{k} \times \mathrm{k}}$, A positive definite, $\mathrm{P}$ orthogonal,

$$
\begin{aligned}
& P^{\prime} A P=\wedge \text { diagonal, } \lambda=\min \wedge(\text { ij) }, 0<\beta<2 \lambda a, \\
& a_{n}=a n^{-1}, c_{n}=c^{-\gamma}, \gamma=\frac{1}{2}(1-\beta) ; \\
& x_{n}-\theta_{n} \rightarrow 0 \quad c>\left\|E_{n}\left(d_{n} d_{n}^{\prime}\right)-\Sigma\right\| \rightarrow 0, \\
& n^{-1} \sum_{j=1}^{n} \alpha_{j, r}^{2} \rightarrow 0 \text { for every } r>0 \text { with } \\
& \sigma_{j, r}^{2}=E_{x}\left\{\left\|d_{j}\right\|^{2} \geq r j^{\alpha}\right\}\left\|d_{j}\right\|^{2} \text { and }
\end{aligned}
$$

let for $x_{n}$ in a neighborhoud of $\theta_{n}$

$$
\begin{aligned}
& \left\|\bar{D}_{n}-A\left(x_{n}-\theta_{n+1}\right)-n^{-\beta / 2} m\right\| \leq 0(1)\left[n^{-\beta / 2}+\left\|^{*} x_{n}-\theta_{n+1}\right\|\right] \\
& \left\|g_{n}(x)-g_{n}\left(\theta_{n}\right)-\left(x_{n}-\theta_{n}\right)\right\| \leq O(1)\left[n^{-\beta / 2}+n^{-1}\left\|x_{n}-\theta_{n}\right\|\right]
\end{aligned}
$$


Then the asymptotic distribution of $n^{\beta / 2}\left(x_{n}-\theta_{n}\right)$ is normal with mean $-a(a A-(\beta / 2) I)^{-1} m$ and covariance matrix PMP' with $M^{(i j)}=a^{2} c^{-2}\left[P^{\prime} \Sigma P\right](i j) /\left(a \wedge(i j)+a \wedge^{(i j)}-\beta\right)$. Proof: From (2.1) (2.2) and (2.3) we have

$$
x_{n+1}-\theta_{n+1}=\stackrel{*}{x}_{n}-\theta_{n+1}-a n^{-1} D_{n}-a n^{-1} d_{n}
$$

From (2.5) it follows that

$$
\bar{D}_{n}=A_{n}\left(\stackrel{*}{x}_{n}-\theta_{n+1}\right) n^{-\beta / 2} m_{n}
$$

with $A_{n}, m_{n}$ are $\not_{n}$-measurable and $A_{n} \rightarrow A, \quad m_{n} \rightarrow m$ uniformally. Thus (5.4) can be written as

$$
\begin{aligned}
x_{n+1}-\theta_{n+1}=\left(\stackrel{x}{x}_{n}-\theta_{n+1}\right)\left(I-a n^{-1} A_{n}\right)-a n^{-1} n^{-\beta / 2} m_{n} \\
-a n^{-1} d_{n}
\end{aligned}
$$

Also it follows from (5.3) that

$$
\stackrel{*}{x}_{n}-\theta_{n+1}=G_{n 1}\left(x_{n}-\theta_{n}\right)+n^{-\beta / 2} g_{n}
$$

where

$$
\begin{aligned}
& \mathrm{G}_{\mathrm{n}} \longrightarrow \mathrm{I} \\
& \mathrm{g}_{\mathrm{n}} \longrightarrow 0
\end{aligned}
$$

uniformally, $\left\|G_{n}-I\right\|=0\left(n^{-1}\right)$.

substituting $(5.6)$ in $(5.5)$ we get

$$
\begin{aligned}
x_{n+1}-\theta_{n+1}= & \left(I-a n^{-1}\left(A_{n}+O(1)\right)\left(x_{n}-\theta_{n}\right)-\right. \\
& a n^{-1} n^{-\beta / 2}\left(m_{n}+0(1)\right)-a n^{-1} d_{n}
\end{aligned}
$$

From (5.7) and Theorem (2.2) Fabian [5], giving the desired result. 


\section{REFERENCES}

[1] Doob, J.L. (1953), Stochastic processes. Wiley, New York.

[2] Dupac, V. (1965), A dynamic stochastic approximation method. Annal. Math. Statist., 1695-1702.

[3] Dupac, V. (1966), Stochastic aproximation in the presence of the trend. Czechosloak Mathematical Jounal,16, 454-461.

[4] Fabian, V. (1968 a), on the choice of design in stochastic approximation methods. Ann. Math. Statist. 39(457-465).

[5] Fabian, V. $(1968$ b), on asymtotic normality in stochastic approximation. Ann. Math. Statist. 39 1327-1332.

[6] Kiefer, J. and Wolfowitz, J. (1952), Stochastic estimation of the maximum of a regression function, Ann. Math. Statist. 23, $462-466$.

[7] Robbins, H. and Monro (1951), A stochastic approximation method. Ann, Math. Statist. 22, 400-407.

[8] Robbins, H. and Siegmund, D. (1971), A convergence theorem for non negative almost super martingles and some applications. In optimizing Methods in statistics (J.S. Rustagi, ed.) 233-257.

[9] Rupper, D. (1985), A Newton-Raphson Version of the multivariate Robbins-Monro procedure. Ann. Statist. 13, 236-245.

[10] Sorour, E. (1978), on the convergence of the dynamic stochastic approximation method for stochastic non-linear multidimensional dyramic dystems. Kybernetika, volume 14 , $28-37$.

[11] Sorour, E. (1991), A dynamic Kiefer-Wolfowitz stochastic approximation procelure. Statistics \& Decision 9, 201-211.

[12] Sorour, E. (1993), Dynamic multivariate stochastic approximation usiry imultaneous perturbation gradient approximation. proceedings of Eighteenth (18) international conference for statistics and computer science and applications (17-22

April), pages ( 13 )
[13] Uosaki, K. (1974), some generalization of the dynamic
stochastic approximation process. Ann. Statist. 2 , 1042-1048. 